\title{
Oscillatory and stationary convective patterns in a reaction driven gravity current
}

Orsika Miholics, ${ }^{1}$ Tamás Rica, ${ }^{1}$ Dezső Horváth, ${ }^{1}$ and Ágota Tóth ${ }^{1,}$ a)

Department of Physical Chemistry and Materials Science, University of Szeged, Aradi vértanúk tere 1., Szeged, H-6720, Hungary

(Dated: 18 October 2011)

Horizontally propagating chemical fronts are studied in a thin solution layer of the acid-catalyzed chlorite-tetrathionate reaction. Unusual cellular patterns develop when significant amount of autocatalyst is bound to polyelectrolyte with low mobility: both oscillatory and stationary patterns evolve as a result of the interaction between the reaction front and the superposed gravity current. The concentration of the polyelectrolyte regulating the velocity of front propagation serves as a bifurcation parameter for switching between the two basic patterns.

a)E-mail: atoth@chem.u-szeged.hu 


\section{INTRODUCTION}

Fluid motion in nature is abound, since density gradients due to composition or temperature change are frequently present. ${ }^{1}$ Convection therefore can be observed as a result of thermal gradients like a rising plume of hot air, mantle convection or different types of clouds. Spreading of pollutants in the ocean or in the atmosphere is mainly due to compositional changes. Buoyancy also plays an important role during cooling of molten metals or carbon dioxide sequestration in brine aquifers. ${ }^{2}$

Chemical reactions coupled with buoyancy driven convection can induce various instabilities. ${ }^{1,3-8}$ The introduction of hydrodynamic motion enhances the local mixing of chemicals at the interface separating the reactants from the products and hence allows for a larger yield of reaction than in the absence of convection. In the simple $\mathrm{A}+\mathrm{B} \longrightarrow \mathrm{C}$ reaction significant enhancement is observed with asymmetric patterns which change in time, ${ }^{9,10}$ since the reaction slows down because of the growing separation of the reactants from the products. ${ }^{11}$ Reactions with positive feedback, like autocatalytic systems, will build a thin interface where the conversion of reactants into products containing the autocatalyst takes place. ${ }^{6,12,13}$ The advantage of these reactions is that along the interface a constant density stratification develops in time, which in turn allows the long-time characterization of the evolving structure with its enhanced local mixing.

During a reaction in a spatially extended system, chemical composition changes result in a change in density which in turn may trigger convection. ${ }^{3}$ In autocatalytic reactions, typically heat is released locally in the course of the reaction leading to density decrease due to volume expansion. The sum of these two contributions will determine how hydrodynamic motion affects the pattern formation. In the presence of buoyancy, an initially planar reaction front may lose stability in the direction perpendicular to the front, ${ }^{6,12,14}$ since the mixing of the reactant is enhanced providing an increase in front velocity, and a cellular structure may evolve if the gravity field is parallel to that of the front propagation. In case of reactions where density decreases, the solutal and thermal effects strengthen each other, ${ }^{5,15}$ while in cases with increasing density, the two compete and even a change in the sign of net density gradient is possible yielding characteristically different patterns. ${ }^{16,17}$ When the two directions are perpendicular, in a vertical slab for example, horizontally propagating vertical planar fronts are always unstable giving rise to a gravity current. ${ }^{18,19}$ 
The acid-catalyzed reaction of the chlorite oxidation of tetrathionate in a slight chlorite excess (CT reaction) is highly exothermic and the solutal density change is positive, i.e., the products are denser than the reactants. ${ }^{7}$ In a sufficiently wide system, the downward propagating fronts are always hydrodynamically unstable, while in thin solutions the upward propagating fronts are stable and retain their initial planar geometry when fronts propagate parallel to the direction of the gravity field. ${ }^{20}$

Lateral instability can also be induced in the absence of convection ${ }^{21,22}$ when the flux of the reactant is significantly greater than that of the autocatalyst and the autocatalytic feedback is sufficiently strong, i.e., the front is characterized as a "pushed type". ${ }^{23}$ The effective diffusion of the autocatalyst can be lowered by reversibly removing an appropriate amount of the autocatalyst utilizing complex formation. In the $\mathrm{CT}$ reaction, carboxylate containing polyacrylamide hydrogels were successfully introduced into the reaction matrix to produce cellular patterns. ${ }^{24-26}$ When a soluble linear polymer is used for binding the autocatalyst, fluid motion due to gravity is still possible therefore both convective and diffusive instabilities arise. ${ }^{27-30}$ The contribution and interaction of these two types of instabilities depend on the extent of autocatalyst binding and the viscosity of the solution. The latter is especially important in matching the time scales associated with each driving force.

We have found experimentally that the use of linear polyacrylamide incorporated with carboxylate groups allows macroscopic fluid motion which, in turn, will induce cellular patterns as the result of convection at a reaction front propagating horizontally in a thin layer of solution. In this manuscript we are going to show that unique oscillatory and stationary patterns can develop in thin solution layers.

\section{EXPERIMENTAL}

Reagent-grade chemicals (Sigma, Aldrich, Reanal) of the CT reaction were used except for $\mathrm{NaClO}_{2}$, which was recrystallized twice to achieve at least $95 \%$ purity. ${ }^{31}$ A $0.30 \mathrm{M}$ sodium polyacrylate stock solution - to bind the hydrogen ion produced in the course of the reaction - was prepared by measuring out sodium hydroxide and polyacrylic acid (100 kDa, Aldrich) in a 1:1 molar ratio. The reactants were then mixed at room temperature yielding the solution composition summarized in Table I, and injected into a Hele-Shaw cell ${ }^{32}$ which was a $16 \mathrm{~cm}$ wide, $2 \mathrm{~mm}$ thick, and $12 \mathrm{~cm}$ long reaction vessel with two $8 \mathrm{~mm}$ thick Plexiglas 
walls as shown in Fig. 1. The cell was positioned horizontally and planar fronts were initiated electrochemically by applying a $2.8 \mathrm{~V}$ potential difference between two Pt wires $(0.25 \mathrm{~mm}$ in diameter) for $30 \mathrm{~s}$. The original basic reactant solution is purple as a result of the applied $\mathrm{pH}$-indicator which changes to yellow in the course of the reaction as the mixture turns acidic. We monitored the traveling reaction fronts from above by a color charge-coupled device camera (SONY DFW-X710) attached to the IEEE 1394 interface of a computer and frames of $800 \times 600$ pixels were digitized in preset $1-5 \mathrm{~s}$ intervals.

The evolving front structure was characterized by its velocity and wavelength. At each time, the front position was defined as the point of inflection in the gray scale values along the direction of propagation ( $x$ coordinate). The mean front position was determined by averaging all the front positions perpendicular to the direction of the propagation ( $y$ coordinate). From the time evolution of the front position, the front velocity was calculated as the slope of the linear regime. The wavelength of the spatially periodic pattern was obtained by calculating the spatial autocorrelation according to

$$
R_{j}=\frac{\sum_{i=1}^{N-j}\left(x_{i}-\bar{x}\right)\left(x_{i+j}-\bar{x}\right)}{\sum_{i=1}^{N}\left(x_{i}-\bar{x}\right)^{2}}
$$

where $x_{i}$ is the front position, $\bar{x}$ is the mean front position and $N$ is the number of points perpendicular to the direction of propagation. Knowing the spatial resolution $\Delta y, R_{j}$ was converted to its dimensional form as $R_{y}=\Delta y R_{j}$.

The density of the reactant and the product solution was measured by an AP Paar DMA 58 digital densitometer within $10^{-5} \mathrm{~g} / \mathrm{cm}^{3}$ precision. In selected experiments temperature in the vicinity of the reaction front was monitored by a type $\mathrm{J}$ thermocouple $(0.5 \mathrm{~mm}$ thin) positioned in the middle of the Hele-Shaw cell.

\section{RESULTS \& DISCUSSION}

The propagation of initially planar, vertically oriented fronts in the horizontal direction is investigated. Although the height of the solution layer is only $2 \mathrm{~mm}$, fluid motion in the vertical direction arises under the experimental conditions, since density increases in the course of the reaction under isothermal conditions, i.e., the product solution is denser than the reactant $\left(\Delta \rho=2.1 \times 10^{-4} \mathrm{~g} / \mathrm{cm}^{3}\right) .{ }^{30}$ This results in a gravity current superimposed on 
the chemical front as the denser product solution tends to advance on the bottom in the form of a single convection roll, and the reaction front takes on a steady geometry, which appears planar upon viewing from above. Sodium polyacrylate in the reactant mixture, however, binds a substantial amount of the autocatalyst hydrogen ion resulting in a significant decrease of the propagation velocity from the value of $7.8 \mathrm{~mm} / \mathrm{min}$ obtained in the absence of polyacrylate. ${ }^{31}$ As the reaction front slows down upon the addition of the polyelectrolyte, thermal effects cannot be neglected any more. In the exothermic CT reaction with our experimental conditions, the maximum temperature rise is $0.8^{\circ} \mathrm{C}$ indicating that the local density decrease due to the thermal expansion of the solution contributes to the resultant density change at the reaction front. It is important to point out, that sodium polyacrylate is a bulky species with diffusion coefficient at least an order of magnitude lower than the other chemical components present in the system, ${ }^{30}$ which would lead to diffusive instabilities in a convection free environment. By comparing the time scale of diffusive patterns (hour scale) ${ }^{25}$ to that of our patterns (minute scale), it is evident that hydrodynamic motion is the dominant form of transport and hence diffusion-driven instability may be neglected.

When the sodium polyacrylate content of the reactant mixture is raised to $25 \mathrm{mM}$, the level where $83 \%$ of the hydrogen ion produced during the reaction is bound to the polymer, the steady front propagation with a single convection roll is not stable any more. Top views of the evolving front are presented in Fig. 2, which illustrate that the pattern has a distinct three-dimensional structure. Close inspection reveals that the leading blurred line in Figs. 2 (a), (f), and (h) that separates the reactant from the product lies on the bottom of the solution layer. On the other hand, the trailing, sharp, very bright cellular structure appears at the upper layers of the solution. The darker straight lines ending at the cusps of the cells locate areas of downward moving fluid in the vicinity of the front, and hence support that convection rolls develop not only along the front but also perpendicular to that. The cells evolve periodically by rising from below just beyond the leading edge of the front where the conversion of reactants into products is high. Since thermal contribution is significant, the warmer product solution can ascend because its density is lowered below that of the reactant yielding a symmetric pattern wedged between the reactant and product zone, that resembles the hexagonal structure in Rayleigh-Bénard instability.

Both the spatial pattern and its temporal evolution are periodic. The time evolution of the structure can be monitored by following the position of the cusps. Figure 3 shows 
that the structure is shifted in $\sim 70-80 \mathrm{~s}$ time intervals by a half wavelength in lateral direction (along the $y$ coordinate). Within these intervals, however, the position of the structures is stationary and their movement along the front is negligible, indicating that vertical fluid motion dominates within the front zone: ascending in the light yellow region and descending in the dark purple region. The wavelength associated with the spatial structure can be obtained from the correlation function depicted by a solid line in Fig. 4 . The first local maximum represents the wavelength of the structure around $\lambda=1.36 \mathrm{~mm}$. The temporal invariance of this local maximum suggests that the pattern is sufficiently stable, since the characteristic wavelength remains $1.37 \pm 0.02 \mathrm{~mm}$ in a $300 \mathrm{~s}$ interval, i.e., over several cycles. Although the pattern itself switches periodically, it travels at a constant speed of $1.32 \pm 0.02 \mathrm{~mm} / \mathrm{min}$, which is significantly greater than that measured in $0.8 \mathrm{~mm}$ thin solution layer with similar experimental conditions $\left(0.787 \pm 0.002 \mathrm{~mm} / \mathrm{min}^{30}\right)$. The increase in front velocity with solution height indicates the local mixing of reactants and products is stronger due to the enhanced convective motion.

On further increasing the concentration of sodium polyacrylate to $27.5 \mathrm{mM}$-corresponding to $92 \%$ binding of the autocatalyst - a distinctly different cellular structure is observed as depicted in Fig. 5. The leading edge of the front is still planar from above, similarly to the previous pattern (cf. Fig. 2), and it develops at the bottom of the Hele-Shaw reaction container. The trailing structure behind is spatially periodic but its wavelength is significantly smaller. The parallel dark purple stripes ending at the cusps of the cells are wider suggesting stronger convection perpendicular to the direction of front propagation.

The front characterized with this comb pattern travels without any significant change in the lateral direction. The cusp evolution, presented in Fig. 6, also underlies that the structure is stationary, since the lateral position of cusps remains constant in time. From the correlation function shown by a dashed line in Fig. 4, the wavelength is $0.78 \pm 0.05 \mathrm{~mm}$ which is approximately half of the wavelength of the oscillatory pattern, therefore the bifurcation from oscillatory to stationary front pattern is accompanied by wavelength halving. Since greater amount of hydrogen ion is bound to the polyelectrolyte and hence less free autocatalyst is present in the system, the constant front velocity $(1.09 \pm 0.02 \mathrm{~mm} / \mathrm{min})$ is slightly lower than in the previous case.

The superposition of the former two patterns illustrated in Fig. 7 is observed when the polyelectrolyte concentration is set to $c=28.1 \mathrm{mM}$ : in the upper solution layer it resembles the 
oscillatory pattern, while in the lower layer the stationary structure with smaller wavelength is visible. The correlation function, shown by a dotted line in Fig. 4, clearly illustrates this dual feature, since it has two fundamental local maxima: the first one at $0.77 \mathrm{~mm}$ and the second one with larger correlation at $1.55 \mathrm{~mm}$.

In this work, we have shown that a significant decrease in the velocity of propagation of a chemical front, accomplished by reversible binding of the autocatalyst into a polyelectrolyte with low mobility, leads to three dimensional convective patterns even in thin horizontal solution layers. The slowing down of the reaction enhances the thermal contribution to the density change in the course of the reaction, due to the exothermicity of the autocatalytic process driving the front. The emerging interaction of the reaction front propagating horizontally and the coexisting gravity current establishes a narrow zone where three dimensional patterns resembling those in Rayleigh-Bénard instability arise. The top views of these structures, envisioned by the aid of a $\mathrm{pH}$ indicator, reveal that in the coordinate system traveling with the reaction front oscillatory and stationary modes of fluid motion with distinct wavelengths exist. The selection of these individual modes and their superposition can be tuned by the amount of polyelectrolyte added to the reactant mixture, indicating that not only thermal effects but also differential diffusion, with significant difference in the effective diffusion coefficients of the key species, are involved in the driving force of this phenomenon.

\section{ACKNOWLEDGMENTS}

This work was financially supported by the Hungarian Scientific Research Fund (OTKA K72365) and ESA through PECS Experimental Arrangements (4000102255/11/NL/KML). 


\section{REFERENCES}

${ }^{1}$ I.R. Epstein and J.A. Pojman, An Introduction to Nonlinear Dynamics: Oscillations, Waves, Patterns, and Chaos, Oxford University Press, Oxford, 1998.

${ }^{2}$ J.T.H. Andres and S.S.S. Cardoso, Phys. Rev. E 83, 046312 (2011).

${ }^{3}$ J.A. Pojman and I.R. Epstein, J. Phys. Chem. 94, 4966 (1990).

${ }^{4}$ J.A. Pojman, A. Komlósi and I.P. Nagy, J. Phys. Chem. 100, 16209 (1996).

${ }^{5}$ M. Böckmann and S. C. Müller, Phys. Rev. Lett. 85, 2506 (2000).

${ }^{6}$ A. De Wit, Phys. Rev. Lett. 87, 054502 (2001).

${ }^{7}$ D. Horváth, T. Bánsági, Jr., Á. Tóth, J. Chem. Phys. 117, 4399 (2002).

${ }^{8}$ A. De Wit, Phys. Fluids 16, 163 (2004).

${ }^{9}$ A. Zalts, C. El Hasi, D. Rubio, A. Ureña, A D’Onofrio, Phys. Rev. E 77, 015304(R) (2008).

${ }^{10}$ C. Almarcha, P.M.J. Trevelyan, P. Grosfils, A. De Wit, Phys. Rev. Lett. 104, 044501 (2010).

${ }^{11}$ L. Gálfi and Z. Rácz, Phys. Rev. A 38, 3151 (1988).

${ }^{12}$ J. Huang, D.A. Vasquez, B.F. Edwards and P. Kolodner, Phys. Rev. E 48, 4378 (1993).

${ }^{13}$ D.A. Vasquez and D.I. Coroian, Chaos 20033109 (2010).

${ }^{14}$ J. Yang, A. D’Onofrio, S. Kalliadasis and A. De Wit, J. Chem. Phys. 117, 9395 (2002).

${ }^{15}$ L. Sebestiková, J.D. D'Hernoncourt, M.J.B. Hauser, S.C. Müller and A. De Wit, Phys. Rev. E 75, 026309 (2007).

${ }^{16}$ T. Bánsági, Jr., D. Horváth, Á. Tóth, J. Yang, S. Kalliadasis and A. De Wit, Phys. Rev. E 68, 055301 (2003).

${ }^{17}$ S. Kalliadasis, J. Yang and A. De Wit, Phys. Fluids 16, 1395 (2004).

${ }^{18}$ L. Rongy, G. Schuszter, Z. Sinkó, T. Tóth, D. Horváth, Á. Tóth, A. De Wit, Chaos 19, 023110 (2009).

${ }^{19}$ G. Schuszter, T. Tóth, D. Horváth and Á. Tóth, Phys. Rev. E 79, 016216 (2009).

${ }^{20}$ T. Bánsági, Jr., D. Horváth and Á. Tóth, J. Chem. Phys. 121, 11912 (2004).

${ }^{21}$ D. Horváth, V. Petrov, S.K. Scott and K. Showalter, J. Chem. Phys. 98, 6332 (1993).

${ }^{22}$ É. Jakab, D. Horváth, Á. Tóth, J.H. Merkin and S.K. Scott, Chem. Phys. Lett. 342, 317 (2001).

${ }^{23}$ W. van Saarloos, Phys. Rep. 386, 29 (2003).

${ }^{24}$ Á. Tóth, I. Lagzi and D. Horváth, J. Phys. Chem. 100, 14837 (1996). 
${ }^{25}$ D. Horváth and Á. Tóth, J. Chem. Phys. 108, 1447 (1998).

${ }^{26}$ M. Fuentes, M.N. Kuperman and P. De Kepper, J. Phys. Chem. A 105, 6769 (2001).

${ }^{27}$ J. D'Hernoncourt, J.H. Merkin and A. De Wit, Phys. Rev. E 76, 035301R (2007).

${ }^{28}$ J. D'Hernoncourt, J.H. Merkin and A. De Wit, J. Chem. Phys. 130, 114502 (2009).

${ }^{29}$ J. D'Hernoncourt, J.H. Merkin and A. De Wit, J. Chem. Phys. 130, 114503 (2009).

${ }^{30}$ T. Rica, É. Pópity-Tóth, D. Horváth and Á. Tóth, Physica D 239, 831 (2010).

${ }^{31}$ Á. Tóth, D. Horváth and A. Siska, J. Chem. Soc., Faraday Trans. 93, 73 (1997).

${ }^{32}$ H.S. Hele-Shaw, Nature 58, 34 (1898). 
TABLE I. Composition of reactant solution where PANa corresponds to sodium polyacrylate

\begin{tabular}{ll}
\hline$\left[\mathrm{NaClO}_{2}\right] / \mathrm{mM}$ & 20.0 \\
{$\left[\mathrm{~K}_{2} \mathrm{~S}_{4} \mathrm{O}_{6}\right] / \mathrm{mM}$} & 5.0 \\
{$[\mathrm{NaOH}] / \mathrm{mM}$} & 1.0 \\
{$[$ Bromophenolblue $] / \mathrm{mM}$} & 0.16 \\
{$[\mathrm{PANa}] / \mathrm{mM}$} & $25.0-29.0$ \\
\hline
\end{tabular}


Fig. 1 Scheme of the Hele-Shaw cell. The propagation of reaction fronts in the horizontal cell is monitored from above.

Fig. 2 Images of the fronts at $\Delta t=10 \mathrm{~s}$ intervals with $[\mathrm{PANa}]=25.0 \mathrm{mM}$. The field of view: $4.5 \times 6.0 \mathrm{~mm}$. Darker purple regions represent the reactant solution and the lighter yellow ones the product. The camera position is shifted in the $x$ direction for the last figure in order to emphasize the oscillatory nature of the front. White arrow points to the leading blurred line, and white dashed line indicates the trailing structure. The white hexagons are drawn to aid the eye to the pattern remnant of the hexagonal symmetry.

Fig. 3 Time evolution of the position of cusps in Fig. 2.

Fig. 4 The spatial autocorrelation function $\left(R_{y}\right)$ for the pattern in Fig. 2 (solid line), in Fig. 5 (dashed line), and in Fig. 7 (dotted line).

Fig. 5 The image of the stable front with $[\mathrm{PANa}]=27.5 \mathrm{mM}$. The field of view: $5.2 \times$ $7.0 \mathrm{~mm}$. Darker purple regions represent the reactant solution and the lighter yellow ones the product.

Fig. 6 Time evolution of the position of cusps in Fig. 5.

Fig. 7 The image of the evolving pattern with $[\mathrm{PANa}]=28.1 \mathrm{mM}$. The field of view: 5.2 $\times 7.0 \mathrm{~mm}$. Darker purple regions represent the reactant solution and the lighter yellow ones the product. 


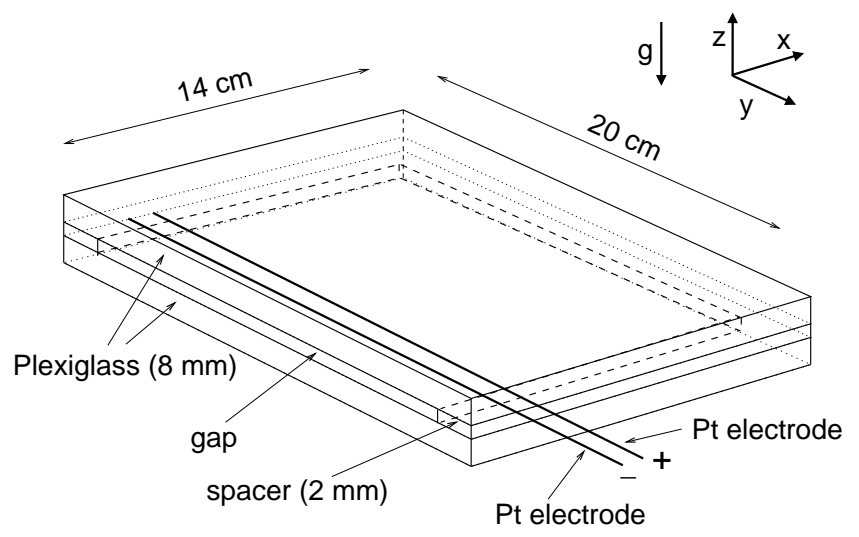

FIG. 1. O. Miholics .... J. Chem. Phys. 

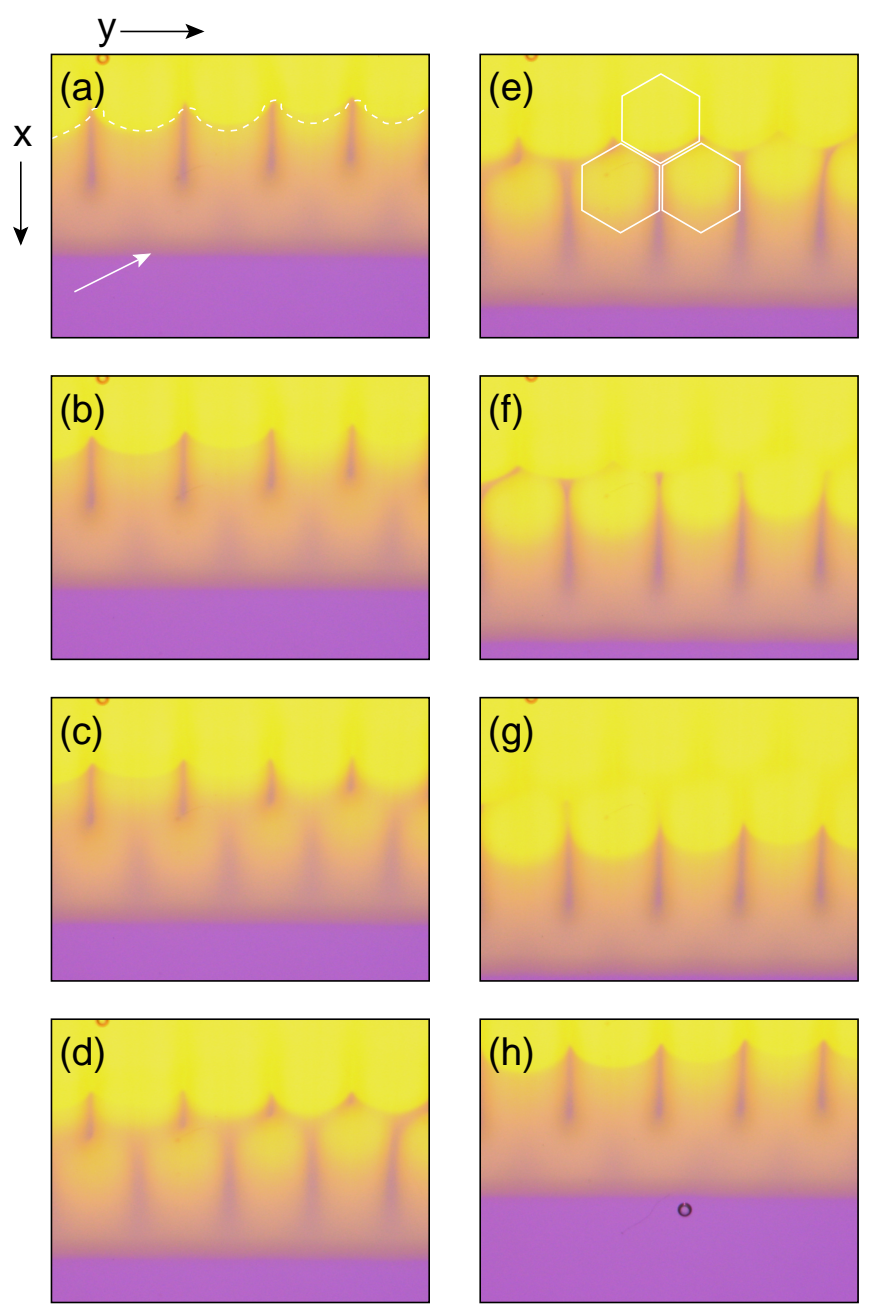

FIG. 2. O. Miholics .... J. Chem. Phys. 


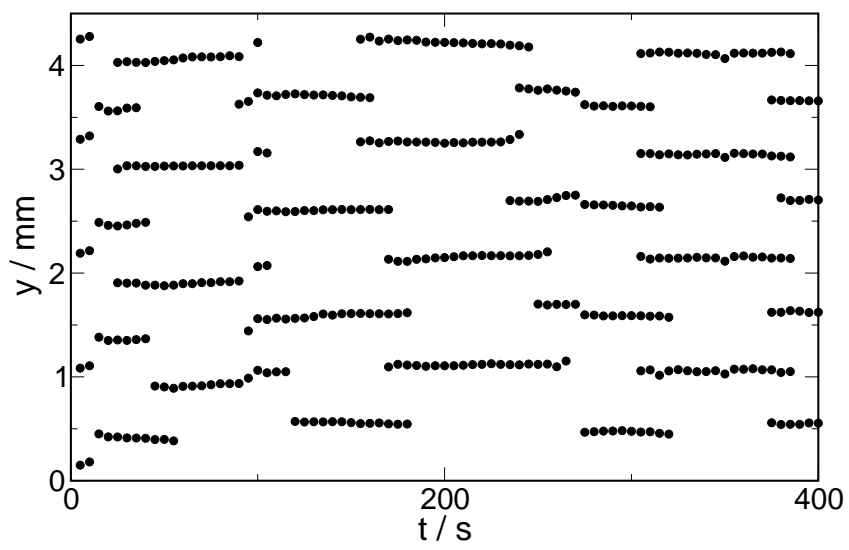

FIG. 3. O. Miholics .... J. Chem. Phys. 


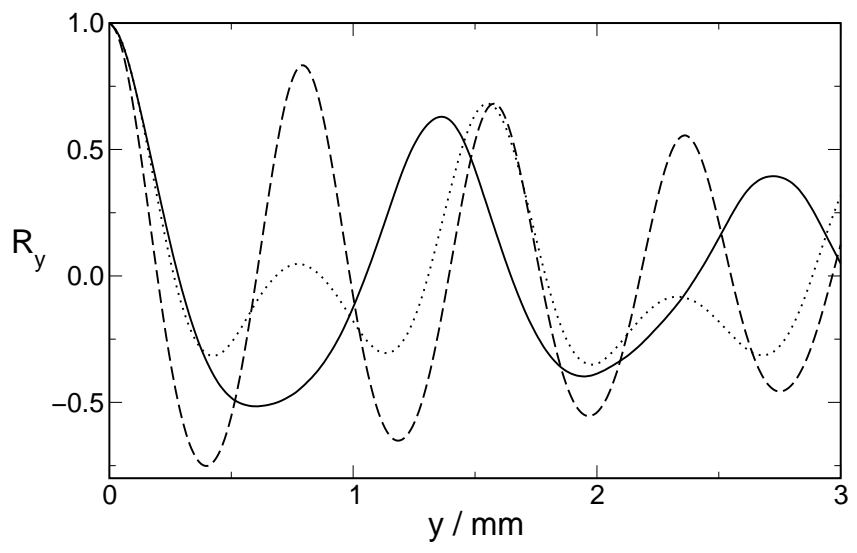

FIG. 4. O. Miholics .... J. Chem. Phys. 


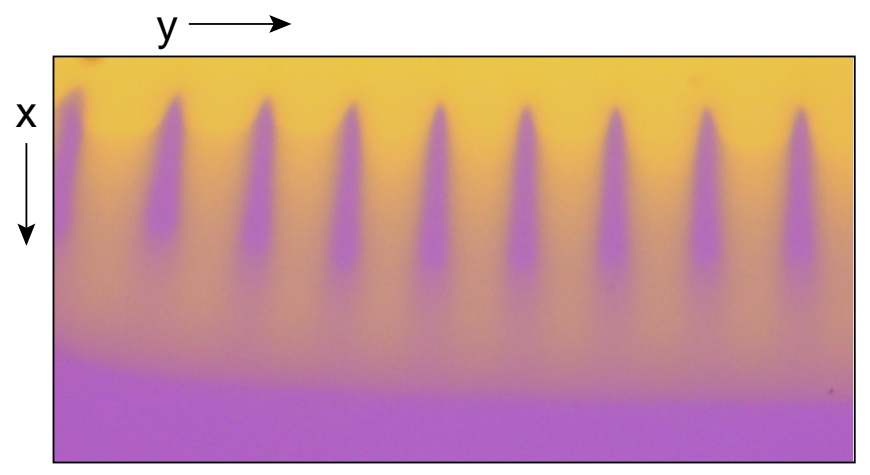

FIG. 5. O. Miholics .... J. Chem. Phys. 


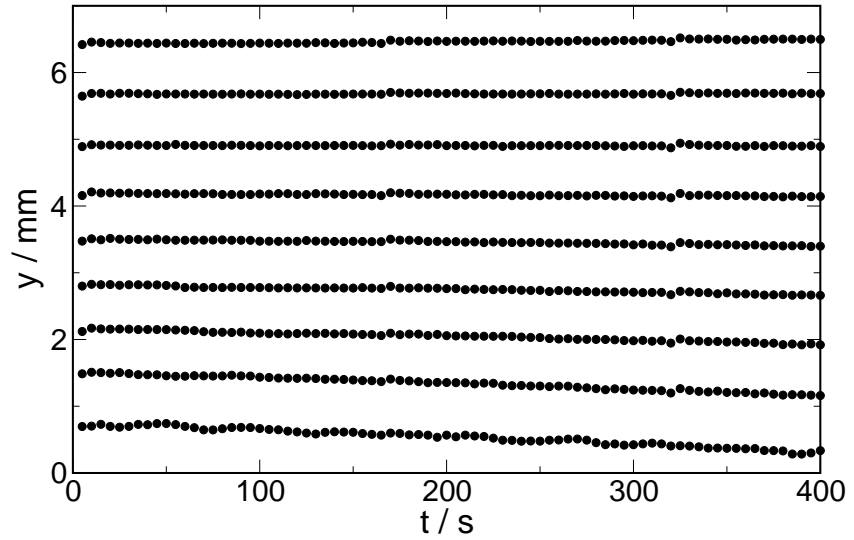

FIG. 6. O. Miholics .... J. Chem. Phys. 


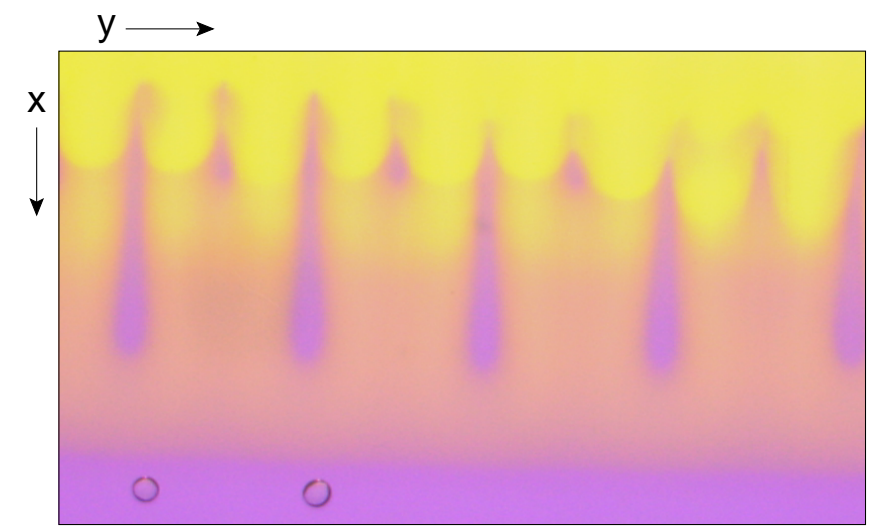

FIG. 7. O. Miholics .... J. Chem. Phys. 